\title{
Recidiva local tras cirugía del cáncer de pulmón
}

Jarabo Sarceda JR, Torres García AJ. Recidiva local tras cirugía del cáncer de pulmón. An Med Interna (Madrid) 2008; 25 : 53-54.

El carcinoma broncogénico es el principal causante de muerte por cáncer. La resección quirúrgica continúa siendo un pilar insustituible en el tratamiento con intención curativa de esta enfermedad, aunque su papel hoy por hoy se restringe a estadios precoces. Y sin embargo hasta la mitad de pacientes resecados en estas condiciones desarrollan recaídas locales o a distancia que a la postre resultarán letales. Identificar factores que nos ayuden a definir qué selección de pacientes resecados son más susceptibles de recaer es fundamental para implementar terapias adyuvantes más agresivas o incluso seguimientos más exhaustivos con el fin de (en lo posible) prevenir, detectar, y en su caso tratar las recidivas. En la literatura se han analizado múltiples factores clínicos (tanto del paciente como del tumor), patológicos y quirúrgicos. La clasificación TNM en sus sucesivas versiones (para 2009 se prevé una nueva actualización basada en los estudios de los últimos años) integra aquellos factores que con mayor evidencia establecen grupos pronósticos más o menos homogéneos (1). Pero existen otros parámetros no incluidos en dicha clasificación que han demostrado estar relacionados con el devenir de la enfermedad y el riesgo de recidiva tras la resección quirúrgica.

Además, en los últimos años, se ha abierto ante nosotros la puerta de un mundo muy complejo: el comportamiento biológico tumoral, a nivel proteico y biomolecular. Es un aspecto poco conocido hasta ahora y con elementos todavía no aplicables de rutina en la práctica clínica, pero que sin duda multiplicará la precisión en la valoración pronóstica de esta enfermedad:

Si nos centramos en las recidivas locales, un paciente con un carcinoma broncogénico no microcítico resecado tiene per se una posibilidad de recaer más o menos definida por distintos factores de riesgo demostrados hasta el momento. Factores clínicos: en varios estudios variables como la edad, el género o el estado funcional del paciente actuaron como factores de riesgo de recidiva. Algunas revisiones amplias, como la basada en el California Cancer Registry, encuentran que el nivel socioeconómico, la edad avanzada al diagnóstico y el género masculino influyeron en la supervivencia por recidiva en pacientes resecados en estadio I (2). Estudios recientes parecen demostrar que pacientes con enfermedad pulmonar obstructiva crónica tienen más riesgo de recidiva tras resección de tumores en estadios iniciales (3). De forma indirecta la situación funcional respiratoria puede determinar resecciones más conservadoras que también han demostrado mayor incidencia de recidivas. Factores radiológicos: Parámetros como la imagen de retracción pleural, la lobulación o la ausencia de broncograma aéreo en la tomografía computarizada se han relacionado con menor intervalo libre de enfermedad en estadio I (4). Factores analíticos: Marcadores tumorales séricos como al antígeno carcinoembrionario, el antígeno de células escamosas o la enolasa neuronal específica parecen influir en el devenir de estos pacientes, como ocurre en otros tipos de tumores (5). Factores relacionados con el tumor: Además de las variables incluidas en la estadificación TNM, otras variables permiten predecir el comportamiento de la enfermedad una vez resecado el tumor. Es el caso de la afectación de la pleura visceral, como en el trabajo presentado en este número. Pero también han demostrado tal asociación con el pronóstico el tipo histológico, el grado de diferenciación, y ciertos marcadores biomoleculares $(6,7)$. Distintos patrones de expresión proteica y las llamadas "firmas génicas" harán en un futuro razonablemente cercano que cada tumor ante el que nos enfrentemos sea único, como también su pronóstico, y quién sabe, quizá su tratamiento, en eso que vienen a llamarse "terapias a la carta" (8). Factores quirúrgicos: La afectación ganglionar mediastínica, como demuestra el trabajo del equipo del Hospital Puerta del Mar (9), continúa siendo un elemento pronóstico muy importante y justifica sin duda una linfadenectomía mediastínica sistemática (es decir, la resección de las adenopatías de todas las regiones mediastínicas accesibles) asociada a la resección pulmonar del carcinoma broncogénico. Algún estudio ha demostrado incluso beneficio en términos de intervalo libre de enfermedad con respecto al muestreo ganglionar mediastínico (10).

Respecto al tipo de resección del tumor primitivo, desde el estudio randomizado clásico de Ginsberg demostrando un aumento en un $75 \%$ en la tasa de recurrencia tras resecciones menores con respecto a lobectomías en estadio I, distintos estudios han confirmado que el tipo de resección puede influir en el riesgo de recidiva local (11). Aunque controvertido 
durante los primeros años, la cirugía toracoscópica para la resección de tumores pulmonares parece que alcanza en los últimos años resultados superponibles al abordaje abierto tradicional (12). Factores relacionados con la adyuvancia: El papel de los tratamientos complementarios a la cirugía, como son la radioterapia y la quimioterapia, en sus múltiples indicaciones, pautas y momentos de aplicación, es otra fuente de estudio de vital importancia en esa integración de la enfermedad oncológica como multidisciplinaria, y desborda los objetivos de esta reflexión. Sí quisiéramos señalar que estos tratamientos cada vez son más individualizados y que las líneas de investigación actuales en oncología médico-quirúrgica del cáncer de pulmón tratan de identificar qué subgrupos de tumores responden mejor a determinadas líneas de tratamiento en función de características fundamentalmente biomoleculares. Sirva como ejemplo el estudio que desarrolla en estos momentos el Grupo Español de Cáncer de Pulmón (GECP)

\section{Bibliografía}

1. De Leyn P, Lartinois D, Van Schil PE, Rami-Porta R, Passlick B, Zielinski $\mathrm{M}$, et al. ESTS guidelines for preoperative lymph node staging for non-small cell lung cancer. Eur J Cardio-Thorac Surg 2007; 32 (1): 1-8.

2. Ou SH, Zell JA, Ziogas A, Antón-Culver H. Prognostic factors for survival of stage I nonsmall cell lung cancer patients: A population-based analysis of 19,702 stage I patients in the California Cancer Registry from 1989 to 2003. Cancer 2007; 110 (7): 1532-41.

3. Sekine Y, Yamada Y, Chiyo M, Iwata T, Nakajima T, Yasufuku K, et al. Association of chronic obstructive pulmonary disease and tumor recurrence in patients with stage IA lung cancer after complete resection. Ann Thorac Surg 2007; 84 (3): 946-50.

4. Yoshino I, Nakanishi R, Kodate M, Osaki T, Hanagiri T, Takenoyama $\mathrm{M}$ et al. Pleural retraction and intra-tumoral air-bronchogram as prognostic factors for stage I pulmonary adenocarcinoma following complete resection. Int Surg 2000; 85 (2): 105-12.

5. Díez M, Torres A, Maestro ML, Ortega MD, Gómez A, Pollán et al. Prediction of survival and recurrence by serum and cytosolic levels of CEA, CA125 and SCC antigens in resectable non-small-cell lung cancer. Br J Cancer 1996; 73 (10): 1248-54.

6. Manac'h D, Riquet M, Medioni J, Le Pimpec-Barthes F, Dujo A, Danel C. Visceral pleura invasion by non-small cell lung cancer: An underrated bad prognostic factor. Ann Thorac Surg 2001; 71 (4): 1088-93.

7. Kobayashi N, Toyooka S, Soh J, Ichimura K, Yanai H, Suehisa H, et al. administrando líneas de adyuvancia diferentes en función de la expresión de BRCA-1 por parte del tumor resecado en pacientes con afectación ganglionar patológica (13). Esto nos conduce de nuevo al campo de la biología molecular como herramienta de predicción de riesgo tras resección del carcinoma broncogénico.

Pero hasta que estos factores sean aplicables en la práctica clínica, habremos de echar mano de otros más cercanos y mensurables con los que contamos actualmente en el día a día, y que son en su mayoría, como ya hemos dicho, clínicos, quirúrgicos y patológicos.

\section{J. R. JARABO SARCEDA, A. J. TORRES GARCÍA}

Servicio de Cirugía 2 y Cirugía Torácica. Hospital Clínico San Carlos. Universidad Complutense de Madrid
Risk factors for recurrence and unfavorable prognosis in patients with stage I non-small cell lung cancer and a tumor diameter of $20 \mathrm{~mm}$ or less. J Thorac Oncol 2007; 2 (9): 808-12.

8. Potti A, Mukherjee S, Petersen R, Dressman HK, Bild A, Koontz J, et al. A genomic strategy to refine prognosis in early-stage non-small-cell lung cancer. N Engl J Med 2006; 355 (6): 570-80.

9. Bernal R, León A, Jaén J, Benítez E, Mateo F. Recidiva local tras la cirugía del cáncer de pulmón no microcrítico. Factores pronósticos. An Med Interna (Madrid) 2008; 25: 55-60

10. De Giacomo T, Venuta F, Rendina EA. Role of lymphadenectomy in the treatment of clinical stage I non-small cell lung cancer. Thorac Surg Clin 2007; 17 (2): 217-21.

11. Ginsberg RJ, Rubinstein LV. Randomized trial of lobectomy versus limited resection for T1 N0 non-small cell lung cancer. Lung Cancer Study Group. Ann Thorac Surg 1995; 60 (3): 615-22.

12. Shiraishi T, Shirakusa T, Hiratsuka M, Yamamoto S, Iwasaki A. Videoassisted thoracoscopic surgery lobectomy for c-T1N0M0 primary lung cancer: Its impact on locoregional control. Ann Thorac Surg 2006; 82 (3): 1021-6.

13. Rosell R, Skrzypski M, Jassem E, Taron M, Bartolucci R, et al (2007) BRCA-1: A Novel Prognostic Factor in Resected Non-Small-Cell Lung Cancer. PLoS ONE 2(11: e1129. doi:10.1371/journal.pone. 0001129 . 